\title{
Chinese-chi and Kundalini yoga Meditations Effects on the Autonomic Nervous System: Comparative Study
}

\author{
Anilesh Dey, D. K. Bhattacharya, D.N. Tibarewala, ${ }^{7}$ Nilanjan Dey, ${ }^{5}$ Amira S. Ashour, ${ }^{6}$ Dac-Nhuong Le, ${ }^{7}$ Evgeniya \\ Gospodinova, ${ }^{7}$ Mitko Gospodinov \\ ${ }^{1}$ Department Electronics and Communication Engineering at The Assam Kaziranga University, India \\ ${ }^{2}$ Department of Pure Mathematics University of Calcutta, India \\ ${ }^{3}$ School of Bioscience \& Engineering at Jadavpur University, India \\ ${ }^{4}$ Department of Information Technology in Techno India College of Technology, India \\ ${ }^{5}$ Computers Engineering Department, Computers and Information Technology College \\ ${ }^{6}$ Faculty of Information Technology, Haiphong University, Vietnam. \\ ${ }^{7}$ Computer Systems Engineering at Institute of Systems Engineering and Robotics of Bulgarian Academy of Sciences, Bulgaria
}

\begin{abstract}
Cardiac disease is one of the major causes for death all over the world. Heart rate variability (HRV) is a significant parameter that used in assessing Autonomous Nervous System (ANS) activity. Generally, the 2D Poincare' plot and 3D Poincaré plot of the HRV signals reflect the effect of different external stimuli on the ANS. Meditation is one of such external stimulus, which has different techniques with different types of effects on the ANS. Chinese Chi-meditation and Kundalini yoga are two different effective meditation techniques. The current work is interested with the analysis of the HRV signals under the effect of these two based on meditation techniques. The 2D and 3D Poincare' plots are generally plotted by fitting respectively an ellipse/ellipsoid to the dense region of the constructed Poincare' plot of HRV signals. However, the 2D and 3D Poincaré plots sometimes fail to describe the proper behaviour of the system. Thus in this study, a three-dimensional frequency-delay plot is proposed to properly distinguish these two famous meditation techniques by analyzing their effects on ANS. This proposed 3D frequency-delay plot is applied on HRV signals of eight persons practicing same Chi-meditation and four other persons practising same Kundalini yoga. To substantiate the result for larger sample of data, statistical Student t-test is applied, which shows a satisfactory result in this context. The experimental results established that the Chi-meditation has large impact on the HRV compared to the Kundalini yoga.
\end{abstract}

Keywords - 2D and 3D Poincaré Plot, 3D Frequency Delay Plot, Hypothesis Testing By Student t-Test.

\section{INTRODUCTION}

$\mathrm{M}$ EDITATION is considered an ancient spiritual practice that has potential benefit on health and well-being $[1,2]$. It is a complex physiological process, which affects neural, psychological, behavioral, and autonomic functions. It is considered as an altered state of consciousness, which differs from wakefulness, relaxation at rest, and sleep [3, 4]. Most of the meditation techniques affect the ANS, thus indirectly regulate several organs and muscles. Accordingly, functions of heartbeat, sweating, breathing, and digestion are controlled by the ANS. Recent studies highlighted the psycho-physiological aspects of meditation and its effect [5-15].

Typically, the HRV is a popular non-invasive tool to assess different conditions of heart [16-19]. Nowadays, it is observed that HRV reflects some psychological conditions [20,21]. The HRV analysis studies the period variation between consecutive heart beats to provide valuable information for the ANS assessment. There are two branches of the ANS, namely i) the sympathetic branch, which increases the heart bits, and ii) the parasympathetic branch, which decreases the heart bits. Thus, the observed HRV is an indicator of the dynamic interaction and balance between these two nervous systems. In the resting condition, both the sympathetic and parasympathetic systems are active with parasympathetic dominance. The balance between both systems is constantly varying to optimize the effect of any internal/external stimuli [22]. Accordingly, the HRV can be significantly affected by physiological state changes and various diseases. Due to the noninvasive character of the HRV, it becomes an attractive tool for the study of human physiological response to different stimuli.

There are a variety of mathematical techniques used to analyze HRV. Peng et al. [23] were interested with the effect of the Chinese Chi and Kundalini Yoga meditation techniques in healthy young adults. It was reported an extremely major heart rate oscillations related to slow breathing during these meditation techniques. The authors applied the spectral analysis along with a new analytic technique based on the Hilbert transform to quantify these heart rate dynamics. The experimental results reported greater oscillations' amplitude during theses meditation compared to the pre-meditation control state and in three non-meditation control groups as well.

Kheder et al. [24] introduced an analysis of HRV signals using wavelet transform (WT). The WT assessment as a feature extraction approach was employed to represent the electrophysiological signals. The authors studied the effect on the ANS system of subjects who did some meditation exercises such as the Chi and Yoga. The calculated detail wavelet coefficients of the HRV signals were used as the feature vectors that represented the signals. Kheder et al. [25] suggested a novel proficient feature extraction technique based on the adaptive threshold of wavelet package coefficients. It is used to evaluate the ANS using the background variation of the HRV signal. The proposed method provided the HRV signal representation in a time-frequency form. This provided better insight in the frequency distribution of the HRV signal with time. The ANOVA statistical test was employed for the evaluation of proposed algorithm.

Consequently, in the current work, the effect of meditation on HRV signals under pre-meditative and meditative states is analyzed. A proposed method is applied [26] for this analysis and thereby distinguishes between two different meditation techniques, namely the 
Chinese chi-meditation and Kundalini yoga. Traditional 2D and 3D Poincaré plots [27-33] with proper delay are constructed for the analysis of the effect of meditation on HRV signals under pre-meditative and meditative states. However, no differences can be visual even by fitting an ellipse/ellipsoid in the respective cases to the cloud region of the Poincare' plot of the HRV signals [34]. Consequently, the signal is analyzed in the frequency domain by transferring the signal from the time domain to the frequency domain using Fast Fourier Transform (FFT) [35]. The notion of three-dimensional (3D) frequency-delay plot [26] is applied. Furthermore, student t-test [36] is performed to substantiate the result for larger sample of data statistical.

The structure of the remaining sections is as follows. Section II included the materials and methods used in the proposed system. Afterwards, the results and discussion are represented in Section III. Finally, the conclusion is depicted in Section IV.

\section{Materials And Methods}

During resting conditions, the RR interval variations characterize a fine tuning of beat-to-beat control. Typically, the HRV signals analysis is very significant for the ANS study to evaluate the stability between the sympathetic and parasympathetic effects on the heart rhythm. Since, the physical activity level is obviously specified in the HRV power spectrum. Thus, the current work proposed a method to effectively analyze the HRV as an indication the ANS system of subjects who are performing meditation exercises such as the Chinesechi and Kundalini yoga.

\section{A. Subjects and Meditation Techniques}

In this study, two popular meditative techniques, namely Chinese Chi (Qigong) meditation and the traditional Kundalini yoga are concerned. All the data are collected from PhysioNet [37]. The Chi meditators were all graduate and post-doctoral students. They were relatively novices in their practice of Chi meditation; most of them began their meditation practice about 1-3 months before this study. All the subjects were healthy, who sign consent in accord with a protocol approved by the Beth Israel Deaconess Medical Centre Institutional Review Board.

Eight Chi meditators, who are 5 women and 3 men (age range 26-35 yrs), wore a Holter recorder for 10 hours during their ordinary daily activities were engaged in this study. During approximately 5 hours into the recording, each of the meditators practiced one hour of meditation. Beginning and ending of meditation times were delineated with event marks. During these sessions, the Chi meditators sat quietly, listening to the taped guidance. The meditators were instructed to breathe spontaneously. The meditation session lasted after about one hour.

For Kundalini Yoga meditation, four meditators (2 women and 2 men: age range $20-52 \mathrm{yrs}$ ), wore a Holter monitor for approximately one and half hours. Fifteen minutes of baseline quiet breathing were recorded before the 1 hour of meditation. The meditation protocol consisted of a sequence of breathing and chanting exercises, performed while seated in a cross-legged posture. The beginning and ending of the various meditation sub-phases were delineated with event marks.

\section{B. Poincaré plots for HRV Analysis}

To explore the HRV dynamics on 'beat-to-beat' basis, the original idea of 2D Poincaré plot included a delay of one beat only with nonunit lag is developed. In order to obtain comparatively better form of 2D Poincaré plot, proper quantification of the 2D Poincaré plot is required for the purpose of interpretation of the behavior of the data. For example, when quantification of 2D Poincaré Plot is performed by the process of 'ellipse fit', then for this ellipse, independent coordinates are required from the data itself. Generally, for quantifying the Poincaré plot, it should not have irregular shape. Hence, it is necessary to select proper lag for constructing best 2D Poincaré plot. Therefore, the minimum auto-correlation method and the Average Mutual Information (AMI) method can be employed for obtaining the proper delay [38]. Since, the HRV signal is nonlinear, thus the AMI method is used to construct the Poincaré Plot as follows.

The AMI method is employed to determine useful delay coordinates for plotting. Suppose $\{x(t)\}_{t=1}^{N}$ is given time series. Given the state of the system $x(t)$, a good choice for the delay $\tau$ is significant to provide maximum new information with measurement at $x(t+\tau)$. For too short delay value, then $x(t)$ is very related to $x(t+\tau)$, thus the plot of the data will stay near the line $x(t)=x(t+\tau)$. For too long delay value, then the coordinates are basically independent, thus no information can be gained from the plot. Therefore, the better choice of the delay $\tau$ can be done by calculating the Mutual information function $I(\tau)$ defined by:

$$
I(\tau)=\sum_{t=1}^{N-\tau} P[x(t), x(t+\tau)] \log \left(\frac{P[x(t), x(t+\tau)]}{P[x(t)] P[x(t+\tau)]}\right)
$$

It was suggested in [38] that the value of the delay, where $I(\tau)$ reaches its first minimum be used for the Poincaré reconstruction as illustrated in Fig.1.

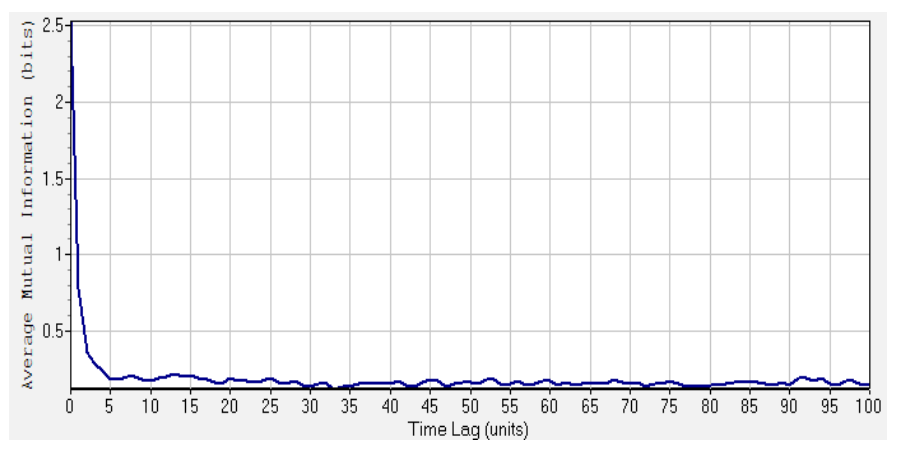

Fig. 1. Graph of the Mutual information function versus the delay

The 2D Poincaré plot is constructed with the independent coordinates $(x(t), x(t+\tau))$ and the 3D Poincaré plot is plotted with the independent coordinates $(x(t), x(t+\tau), x(t+2 \tau))$.

\section{Auto-correlation in frequency domain}

For the auto-correlation process [26], let $\{x(k)\}_{k=1}^{N}$ be the sample of a discrete time signal and $\left\{X(j)=a_{j}+i b_{j} \equiv\left(a_{j}, b_{j}\right)\right\}_{j=1}^{N}$ be its Fourier spectrum. The time series $\{X(j)\}_{j=1}^{N}$ is subdivide into two groups $U=\{X(j)\}_{j=1}^{N-\mu}=\left\{\left(a_{j}, b_{j}\right)\right\}_{j=1}^{N-\mu}$ and $V=\{X(j)\}_{j=1+\mu}^{N}=\left\{\left(a_{j}, b_{j}\right)\right\}_{j=1+\mu}^{N}$ for $\mu=1,2,3,4,5, \ldots \ldots$. 
The autocorrelation of $\{x(j)\}_{j=1}^{N}$ in frequency domain corresponding to lag variable $\mu$ is defined by:

$(\mu)=\frac{\sum_{j=1}^{N}\left|\left\{\left(a_{j}, b_{j}\right)-\left(\bar{a}_{j}, \bar{b}_{j}\right)\right\} \cdot\left\{\left(a_{j+\mu}, b_{j+\mu}\right)-\left(\bar{a}_{j+\mu}, \bar{b}_{j+\mu}\right)\right\}\right|}{\sqrt{\sum_{j=1}^{N}\left|\left\{\left(a_{j}, b_{j}\right)-\left(\bar{a}_{j}, \bar{b}_{j}\right)\right\}\right|^{2}} \cdot \sqrt{\sum_{j=1}^{N} \mid\left\{\left(a_{j+\mu}, b_{j+\mu}\right)-\left(\bar{a}_{j+\mu}, \bar{b}_{j+\mu}\right)\right.}}$

Where, $\left(\bar{a}_{j}, \bar{b}_{j}\right),\left(\bar{a}_{j+\mu}, \bar{b}_{j+\mu}\right)$ are the mean values of $\left\{\left(a_{j}, b_{j}\right)\right\}_{j=1}^{N-\mu}$ and $\quad\left\{\left(a_{j}, b_{j}\right)\right\}_{j=1+\mu}^{N} ;$ respectively. In addition, $\quad\left(a_{r}, b_{r}\right) \cdot\left(a_{s}, b_{s}\right)=\left(a_{r} a_{s}-b_{r} b_{s}, a_{r} b_{s}+b_{r} a_{s}\right)$ for $r, s=1,2,3,4,5, \ldots \ldots . . N$, which called auto-correlation in the frequency domain amongst two stages. In order to define the auto-correlation in the frequency domain amongst three

stages, the time series $\{X(j)\}_{j=1}^{N}$ is subdivided into three groups

$$
\begin{gathered}
U=\{X(j)\}_{j=1}^{N-2 \mu}=\left\{\left(a_{j}, b_{j}\right)\right\}_{j=1}^{N-2 \mu}, V=\{X(j)\}_{j=1+\mu}^{N-\mu}=\left\{\left(a_{j}, b_{j}\right)\right\}_{j=1+\mu}^{N-\mu}, \\
W=\{X(j)\}_{j=1+2 \mu}^{N}=\left\{\left(a_{j}, b_{j}\right)\right\}_{j=1+2 \mu}^{N} \text {. Thus, the auto-correlation }
\end{gathered}
$$

of $\{X(j)\}_{j=1}^{N}$ in frequency domain amongst three stages corresponding to the frequency delay $\mu$ repeated is defined by:

$$
R_{X}(\mu)=\frac{\sum_{j=1}^{N}\left|\zeta_{j} \cdot \zeta_{j+\mu} \cdot \zeta_{j+2 \mu}\right|}{\sqrt{\sum_{j=1}^{N}\left|\zeta_{j}\right|^{2}} \cdot \sqrt{\sum_{j=1}^{N}\left|\zeta_{j+\mu}\right|^{2}} \cdot \sqrt{\sum_{j=1}^{N}\left|\zeta_{j+2 \mu}\right|^{2}}}
$$

$$
\begin{gathered}
\mathrm{W} \quad \mathrm{h} \quad \mathrm{e} \quad \mathrm{r} \text { e }, \quad \mu=1,2, \ldots \ldots \ldots \ldots \ldots,(N-1), \\
\zeta_{j}=\left\{\left(a_{j}, b_{j}\right)-\left(\overline{a_{j}}, \overline{b_{j}}\right)\right\}, \zeta_{j+\mu}=\left\{\left(a_{j+\mu}, b_{j+\mu}\right)-\left(\overline{a_{j+\mu}}, \overline{b_{j+\mu}}\right)\right\} \\
\zeta_{j+2 \mu}=\left\{\left(a_{j+2 \mu}, b_{j+2 \mu}\right)-\left(\overline{a_{j+2 \mu}}, \overline{b_{j+2 \mu}}\right)\right\}
\end{gathered}
$$

and $\left(\bar{a}_{j}, \bar{b}_{j}\right)$ is the mean of $\left(a_{j}, b_{j}\right)$. Moreover,

$$
\left(a_{r}, b_{r}\right) \cdot\left(a_{s}, b_{s}\right)=\left(a_{r} a_{s}-b_{r} b_{s}, a_{r} b_{s}+b_{r} a_{s}\right)
$$

$r, s=1,2,3,4,5, \ldots \ldots . N$ and $\mu=1,2,3,4,5$

In most cases, the signal interpretation in the frequency domain is based on the periodogram (Periodogram analysis), which is framed from the Fourier spectra. Since, a considerable amount of the spectra has to be overlooked or removed during the interpretation of the signals from the corresponding periodogram. Thus, the generality of the frequency domain analysis is lost. To solve this context, Poincaré plot can be used to compare the behaviour of the signal at a given frequency with that at a different frequency in the whole spectrum using analysis similar to what is done in time domain.

\section{The 3D Frequency delay plot and its Quantification}

The 3D frequency delay plot [26] is a plot in 3D space constructed with the independent coordinates $|X(j)|,|X(j+\mu)|,|X(j+2 \mu)|$, where $X(j)$ is the frequency spectrum of the discrete time-signal $X(k)$ obtained by FFT [32] of $X(k)$. The idea is quite similar to that of the $3 \mathrm{D}$ Poincare plot, but as this plot is constructed in the frequency domain with a proper frequency-delay, it is called frequency-delay plot. The proper frequency-delay $(\mu)$ is obtained from the graph of $R_{X(j)}(\mu)$ versus $\mu$ using Eq. (3). In fact, the optimal frequency-delay $(\mu)$ is one for which $R_{X(j)}(\mu)$ comes nearer to zero for the first time. Since, $|X(j)|$ denotes the signal energy, thus the frequency-delay plot gives an insight to the changing energy dynamics of the signal.

Quantification of 3D frequency-delay plot is generally done by ellipsoid method [26]. Since, for most of the signals, the 3D frequencydelay plots are found to be almost dense and well-shaped. Therefore, an ellipsoid having its major axis along the line of identity is fitted to the dense region of the 3D frequency-delay plot. Axes of the ellipsoid stand as a strong indicator of the changing energy dynamics of HRV. Fig. 2 shows the ellipsoid fit to the dense region of the phase space.

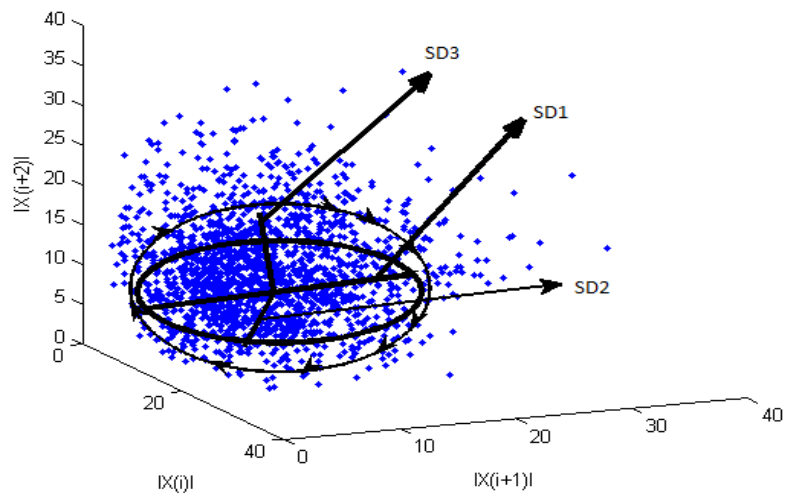

Fig. 2. Ellipsoid fitted on the dense region

Where, SD1, SD2 and SD3 are the axes of the ellipsoid. Let $\{X(j)\}_{j=1}^{N}$ be a discrete signal obtained by applying FFT [35] of the HRV signal. The 3D frequency-delay plot can be constructed by subdividing this signal into three groups as $x^{+}, x^{-}, x^{--}$with the same frequency delay $\mu$, where:

$$
x^{+}=\{|X(j)|\}_{j=1}^{N-2 \mu}, x^{-}=\{|X(j)|\}_{k=1+\mu}^{N-\mu}, x^{--}=\{|X(j)|\}_{j=1+2 \mu}^{N}
$$

Where, $\quad \mu=1,2, \ldots,(N-1)$. The co-ordinate system is transformed by a 3D rotation with same angle $\frac{\pi}{4}$ with respect to $X$
$Y$ and $Z$ axis. The transform is given by: $Y$ and $Z$ axis. The transform is given by:
\[ \left(\begin{array}{l}x_{m} \\ x_{n} \\ x_{p}\end{array}\right)=\left(\begin{array}{ccc}\cos \frac{\pi}{4} \cos \frac{\pi}{4} & \cos \frac{\pi}{4} \sin \frac{\pi}{4} \sin \frac{\pi}{4}-\cos \frac{\pi}{4} \sin \frac{\pi}{4} & \cos \frac{\pi}{4} \cos \frac{\pi}{4} \sin \frac{\pi}{4}+\sin \frac{\pi}{4} \sin \frac{\pi}{4} \\ \cos \frac{\pi}{4} \sin \frac{\pi}{4} & \cos \frac{\pi}{4} \cos \frac{\pi}{4}+\sin \frac{\pi}{4} \sin \frac{\pi}{4} \sin \frac{\pi}{4} & -\cos \frac{\pi}{4} \sin \frac{\pi}{4}+\cos \frac{\pi}{4} \sin \frac{\pi}{4} \sin \frac{\pi}{4} \\ -\sin \frac{\pi}{4} & \cos \frac{\pi}{4} \sin \frac{\pi}{4} & \cos \frac{\pi}{4} \cos \frac{\pi}{4}\end{array}\right)\left(\begin{array}{l}x^{+} \\ x^{-} \\ x^{-}\end{array}\right) \] 


$$
=\frac{1}{2 \sqrt{2}}\left(\begin{array}{ccc}
2 \sqrt{2} & -(\sqrt{2}-1) & (\sqrt{2}+1) \\
2 \sqrt{2} & (\sqrt{2}+1) & -(\sqrt{2}-1) \\
-2 & \sqrt{2} & \sqrt{2}
\end{array}\right)\left(\begin{array}{l}
x^{+} \\
x^{-} \\
x^{--}
\end{array}\right)
$$

Hence,

$$
\begin{aligned}
& x_{m}=\frac{1}{2} \cdot x^{+}+\left(\frac{1}{2 \sqrt{2}}-\frac{1}{2}\right) \cdot x^{-}+\left(\frac{1}{2 \sqrt{2}}+\frac{1}{2}\right) \cdot x^{-}=\frac{2 \sqrt{2} \cdot x^{+}-(\sqrt{2}-1) \cdot x^{-}+(\sqrt{2}+1) \cdot x^{-}}{2 \sqrt{2}} \\
& x_{n}=\frac{1}{2} \cdot x^{+}+\left(\frac{1}{2 \sqrt{2}}+\frac{1}{2}\right) \cdot x^{-}+\left(\frac{1}{2 \sqrt{2}}-\frac{1}{2}\right) \cdot x^{-}=\frac{2 \sqrt{2} \cdot x^{+}+(\sqrt{2}+1) \cdot x^{-}-(\sqrt{2}-1) \cdot x^{-}}{2 \sqrt{2}} \\
& x_{p}=\left(-\frac{1}{\sqrt{2}}\right) \cdot x^{+}+\frac{1}{2} \cdot x^{-}+\frac{1}{2} \cdot x^{--}=\frac{-2 \cdot x^{+}+\sqrt{2} \cdot x^{-}+\sqrt{2} \cdot x^{-}}{2 \sqrt{2}}
\end{aligned}
$$

Thus, a new co-ordinate system $\left(x_{m}, x_{n}, x_{p}\right)$ is formed.

Let $\quad \overline{x_{m}}=\operatorname{Mean}\left(x_{m}\right), \overline{x_{n}}=\operatorname{Mean}\left(x_{n}\right), \overline{x_{p}}=\operatorname{Mean}\left(x_{p}\right)$ and $S D_{1}=\sqrt{\operatorname{Var}\left(x_{m}\right)}, S D_{2}=\sqrt{\operatorname{Var}\left(x_{n}\right)}, S D_{3}=\sqrt{\operatorname{Var}\left(x_{p}\right)} \cdot \quad$ Lastly, $\quad$ an ellipsoid centred at $\left(\overline{x_{m}}, \overline{x_{n}}, \overline{x_{p}}\right)$ with three axes of length $S D_{1}$,

$S D_{2}$ and $S D_{3}$ is taken for quantification of the existing 3D frequency-delay plot.

\section{E. Statistical Hypothesis Test}

Comparison of two populations mean is normally performed by hypothesis testing using Student's t-test [36]. However, the test stands on the assumptions: (i) the populations are normally distributed, and (ii) their variances are homogeneous. Usually the populations are taken to be normally distributed, but the homogeneity of population variances is always to be verified.

\section{Test for equality of the two variances}

Consider the null hypothesis $H_{o}: \sigma_{1}^{2}=\sigma_{2}^{2}$ and the alternative hypothesis $H_{A}: \sigma_{1}^{2} \neq \sigma_{2}^{2}$, where $\sigma_{1}^{2}$ and $\sigma_{2}^{2}$ are the variances.

The test statistic is given by $\mathrm{F}=\frac{\mathrm{s}_{1}^{2}}{\mathrm{~s}_{2}^{2}}$, where $s_{i}^{2}$ are the sample variances. If this calculated value of $\mathrm{F}$ is less than $F_{0.05(2), v_{1}, v_{2}}$; where $v_{1}$ and $v_{2}$ are the degrees of freedom, then $\mathrm{H}_{0}$ holds, otherwise $\mathrm{H}_{\mathrm{A}}$ holds.

Test for equality of two means $\grave{\mathbf{i}}_{1}, \grave{\mathrm{i}}_{2}$ with equal population variances $\mathrm{o}_{1}^{2}=\mathrm{o}_{2}^{2}$

Let the null hypothesis be $\mathrm{H}_{0} \vec{*} \grave{1}_{1} \quad 2$ and the alternate hypothesis is $\mathrm{H}_{A}: \grave{i}_{1} \neq{ }_{2}$. The samples $X_{1}$ and $X_{2}$ with sizes $n_{1}$ and $n_{2}$ are used, where $\overline{\mathrm{X}}_{1}$ and $\overline{\mathrm{X}}_{2}$ are the corresponding sample means. The standard error $\mathrm{S}_{\overline{\mathrm{X}}_{1}-\overline{\mathrm{X}}_{2}}$ is given by:

$$
\mathrm{s}_{\overline{\mathrm{X}}_{1}-\overline{\mathrm{x}}_{2}}=s_{p} \sqrt{\left(\frac{1}{\mathrm{n}_{1}}+\frac{1}{\mathrm{n}_{2}}\right)}
$$

Where, $\mathrm{s}_{\mathrm{p}}$ ariance given by $s_{p}=\frac{\sum_{i} S S_{i}}{\sum_{i} v_{i}}=\frac{\sum_{i} v_{i} s_{i}^{2}}{\sum_{i} v_{i}}$ and $v_{i}$ represents the $i^{\text {th }}$ sample degrees of freedom. The test statistic ' $\mathrm{t}$ ' with degrees of freedom $i_{1}+i_{2}$ is given by:

$$
\mathrm{t}=\frac{\overline{\mathrm{X}}-\overline{\mathrm{Y}}}{s_{p} \sqrt{\left(\frac{1}{\mathrm{n}_{1}}+\frac{1}{\mathrm{n}_{2}}\right)}}
$$

If this calculated value of $\mathrm{t}$ is less than $\mathrm{t}_{\mathbf{0 . 0 5 ( 2 ) ,}{ }_{1} \quad 2}$, then $\mathrm{H}_{0}$ holds, i.e., $\grave{1}_{1}=\grave{i}_{2}$, otherwise $H_{A}$ holds, i.e., $\grave{1}_{1} \neq \grave{i}_{2}$.

From the preceding methodology the Poincare plots with proper delay of HRV signal in pre-meditation and post-meditation states in time domain are employed. Moreover, 3D Frequency-delay plot of HRV signals in pre-meditative and meditative states is represented.

\section{RESULTS AND DiscusSIONS}

The current work is concerned with the HRV analysis to study the effect of the pre-meditation and post-meditation of the Chinese-chi and Kundalini yoga Meditations using time and frequency domain representations.

\section{A. The 2D Poincaré plot with proper delay of HRV signal}

A 2D Poincaré plots with proper delay for the HRV signals of premeditative and meditative states are constructed in the time domain. The proper delay is obtained by the AMI method. Fig. 3 illustrates one such pair of 2D Poincaré plots in pre-meditative and meditative states under Chinese chi meditation.

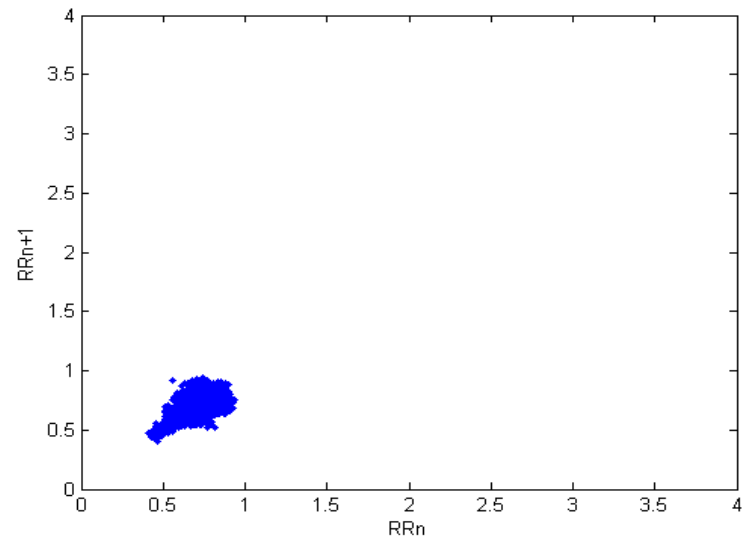

(i) 


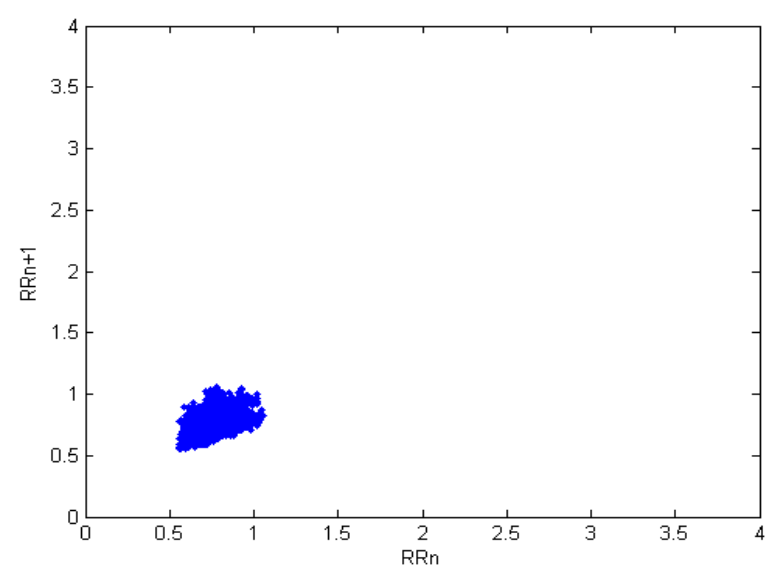

(ii)

Fig.3. The 2D Poincaré Plot with proper delay of HRV signals in (i) premeditative and (ii) meditative state

Fig. 3 illustrates that both the Poincaré plots are almost dense with very few outliers. Essentially, there is no approach to eliminate these outliers of the plots except with manual supervision and visual inspection. Additionally, it is necessary to focus on the main cluster because the important, relevant and necessary information in this context is hidden within the orientation of the main cluster. Thus, these plots are quantified by fitting an ellipse to their main cluster; and compute the lengths of the major and minor axis in each case. Finally, the ratio of two axes is considered as a quantifying parameter. The results of quantification of 2D Poincaré plot of HRV signals in pre-meditative and meditative states under Chinese-chi meditation and Kundalini yoga are summarized in Table I.

TABLE I

QUANTIFICATION TABLE OF 2D POINCARÉ PLOT OF HRV SIGNALS IN PRE-MEDITATIVE AND MEDITATIVE STATES

\begin{tabular}{ccccccc}
\hline \multirow{2}{*}{ Subjects } & \multicolumn{3}{c}{ Pre-meditative States } & \multicolumn{3}{c}{ Meditative States } \\
\cline { 3 - 8 } & \multicolumn{1}{c}{ SD1 } & SD2 & SD2/SD1 & SD1 & SD2 & SD2/SD1 \\
c1 & 0.217168 & 0.248071 & 1.142297 & 0.096461 & 0.092843 & 0.9625 \\
c2 & 0.08111 & 0.17088 & 2.106766 & 0.092317 & 0.091992 & 0.996483 \\
c3 & 0.065666 & 0.168197 & 2.561396 & 0.072398 & 0.085996 & 1.187816 \\
c4 & 0.067267 & 0.177687 & 2.641518 & 0.098595 & 0.10242 & 1.038795 \\
c5 & 0.035275 & 0.085689 & 2.429142 & 0.054112 & 0.066373 & 1.226589 \\
c6 & 0.051249 & 0.095185 & 1.857299 & 0.078813 & 0.103829 & 1.317402 \\
c7 & 0.201171 & 0.224579 & 1.116359 & 0.100892 & 0.123328 & 1.222379 \\
c8 & 0.048568 & 0.106893 & 2.200897 & 0.081619 & 0.09105 & 1.115541 \\
& & KUNDALINI YOGA & & \\
y1 & 0.034221 & 0.050117 & 1.464478 & 0.056986 & 0.067504 & 1.184563 \\
y2 & 0.050891 & 0.087036 & 1.710252 & 0.078341 & 0.065093 & 0.830892 \\
y3 & 0.062703 & 0.078124 & 1.245926 & 0.099425 & 0.079435 & 0.798945 \\
y4 & 0.163102 & 0.235673 & 1.444941 & 0.166584 & 0.15217 & 0.91347 \\
\hline
\end{tabular}

Table I depicts that the ratio of the axis length $\mathrm{SD}_{2} / \mathrm{SD}_{1}$ decreases in meditative states for all subjects except $\mathrm{c} 7$ under Chinese-chi meditation, where the ratio value increases in the meditative states. However, the ratio decreases in meditative state for all subjects under Kundalini yoga. Thus, the 2D Poincaré plot with proper delay is improper tool for distinguishing the two different techniques of meditations. Therefore, the 3D Poincaré plot with proper delay is used instead of the 2D Poincaré plot with proper delay.

\section{B. The 3D Poincaré Plot with proper delay of HRV signal}

The 3D Poincare plots with proper delay for the HRV signals of pre-meditative and meditative states are constructed. The proper delay is obtained by the AMI method as obtained in case of 2D Poincaré plots. Fig. 4 shows a pair of 3D Poincare plots in pre-meditative and meditative states under Chinese-chi meditation.

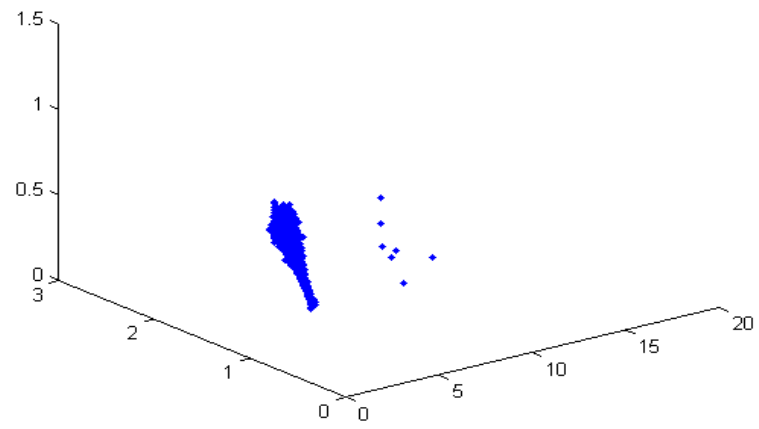

(i)

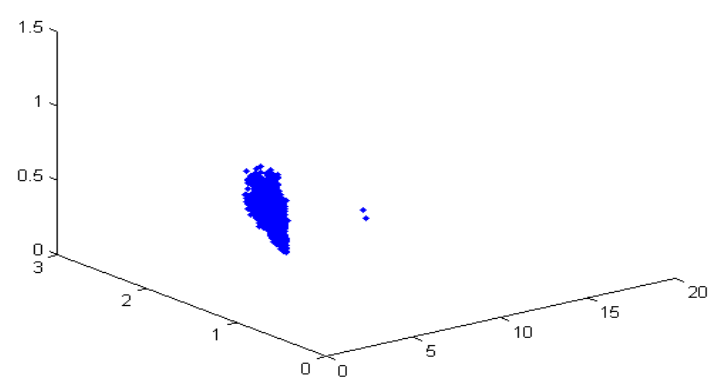

(ii)

Fig. 4. The 3D Poincaré plot with proper delay of HRV signals in (i) premeditative and (ii) meditative state

Fig. 4 establishes that both the plots are well-formed and dense compared to the previously obtained 2D Poincaré plots in premeditative and meditative states. So, these plots are quantified by fitting an ellipsoid to their main clusters. For this purpose, the lengths of three axes SD1, SD2, and SD3 are computed. In addition, R21= $\mathrm{SD} 2 / \mathrm{SD} 1$ and $\mathrm{R} 23=\mathrm{SD} 2 / \mathrm{SD} 3$ are calculated. Finally, the quantifying parameter $(\mathrm{R})$ is identified as the average of the two aforesaid ratios, which given by:

$$
R=\frac{1}{2}\left(\frac{S D_{2}}{S D_{1}}+\frac{S D_{2}}{S D_{3}}\right)
$$

Table II depicts the quantification Table of the 3D Poincaré plot of HRV signals in pre-meditative and meditative states under Chinese-chi meditation and Kundalini yoga.

TABLE II

QUANTIFICATION TABLE OF THE 3D POINCARÉ PLOT OF HRV SIGNALS IN PRE-MEDITATIVE AND MEDITATIVE STATES

\begin{tabular}{|c|c|c|c|c|c|c|c|c|}
\hline \multirow{2}{*}{$\begin{array}{l}\frac{\vec{d}}{0} \\
\frac{\tilde{D}}{\vec{B}}\end{array}$} & \multicolumn{4}{|c|}{ Pre-meditative States } & \multicolumn{4}{|c|}{ Meditative States } \\
\hline & SD1 & SD2 & SD3 & $\mathrm{R}$ & SD1 & SD2 & SD3 & $\mathrm{R}$ \\
\hline
\end{tabular}




\begin{tabular}{lcccccccc}
\multicolumn{8}{c}{ CHINESE - CHI MEDITATION } \\
c1 & 0.319503 & 0.322197 & 0.220053 & 1.236306 & 0.107617 & 0.1262 & 0.103898 & 1.193662 \\
c2 & 0.210006 & 0.214627 & 0.090175 & 1.701052 & 0.131799 & 0.119873 & 0.086545 & 1.147309 \\
c3 & 0.201802 & 0.20643 & 0.078011 & 1.834554 & 0.11065 & 0.11111 & 0.073301 & 1.259989 \\
c4 & 0.211601 & 0.218672 & 0.082219 & 1.846527 & 0.136911 & 0.134332 & 0.09753 & 1.179251 \\
c5 & 0.102699 & 0.105205 & 0.041458 & 1.781009 & 0.088842 & 0.084218 & 0.052217 & 1.280406 \\
c6 & 0.114162 & 0.118252 & 0.057124 & 1.552955 & 0.139055 & 0.130878 & 0.075301 & 1.339627 \\
c7 & 0.290027 & 0.292599 & 0.203594 & 1.223019 & 0.156848 & 0.158954 & 0.10314 & 1.27729 \\
c8 & 0.128552 & 0.132456 & 0.055714 & 1.703893 & 0.121789 & 0.117936 & 0.080072 & 1.22062 \\
& & & KUNDALINI YOGA & & & \\
y1 & 0.064352 & 0.063299 & 0.03404 & 1.421586 & 0.071748 & 0.089372 & 0.065443 & 1.305651 \\
y2 & 0.108028 & 0.1092 & 0.053639 & 1.523349 & 0.096473 & 0.08805 & 0.073817 & 1.052757 \\
y3 & 0.091121 & 0.10185 & 0.069268 & 1.294063 & 0.098497 & 0.111917 & 0.103686 & 1.107814 \\
y4 & 0.301835 & 0.297696 & 0.163431 & 1.403916 & 0.239872 & 0.198262 & 0.145926 & 1.092589 \\
\hline
\end{tabular}

Table II illustrates that the values of $R$ in meditative states are less than that of the pre-meditative states in all the subjects except $\mathrm{c} 7$ under Chinese-chi meditation. However, $R$ decreases in meditative states for all the subjects under Kundalini yoga. Thus, the 3D Poincaré plot with proper delay is improper tool for distinguishing these two different meditation techniques, even it is better than the 2D Poincaré plot. Therefore, frequency domain analysis is to be employed instead of the time domain analysis.

\section{C. $3 D$ Frequency-delay plot of HRV signals in pre-meditative and meditative states}

Each of the HRV signals of pre-meditative and meditative states are transformed into the frequency domain by applying FFT [35] and 3D frequency-delay plots as described in section 2.4. Fig. 5 shows a pair of $3 \mathrm{D}$ frequency-delay plots in pre-meditative and meditative states under Chinese-chi meditation.

Fig. 5 illustrates that all the plots are well-formed and dense compared to the previously obtained 3D Poincaré plots in pre-meditative and meditative states in time domain. So, these plots are quantified by fitting an ellipsoid to their main clusters. For this purpose, the lengths of three axes $\mathrm{SD}_{1}, \mathrm{SD}_{2}$ and $\mathrm{SD}_{3}$ are used to calculate the ratios: $\mathrm{R}_{21}=$ $\mathrm{SD}_{2} / \mathrm{SD}_{1}$ and $\mathrm{R}_{23}=\mathrm{SD}_{2} / \mathrm{SD}_{3}$. Finally, the quantifying parameter $(\mathrm{R})$ is taken as the average of the two aforesaid ratios. Table III summarizes quantification of the 3D frequency-delay plot of HRV signals in premeditative and meditative states under Chinese-chi meditation and Kundalini yoga.

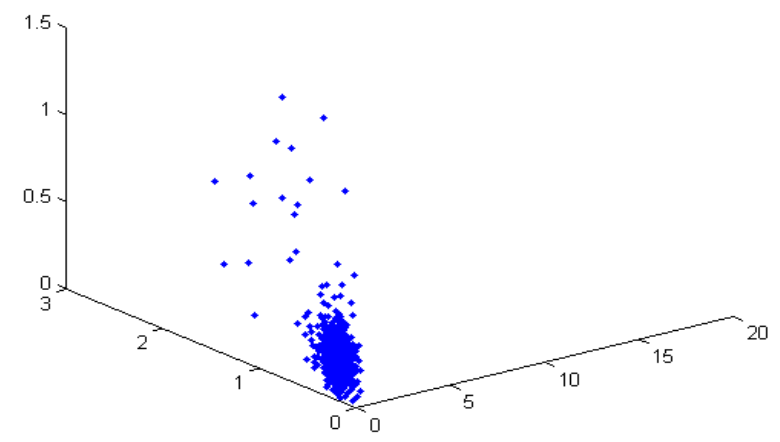

(i)

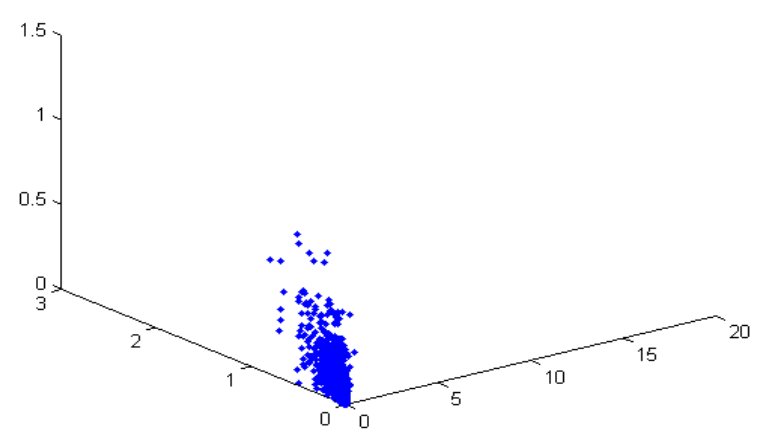

(ii)

Fig. 5. 3D frequency-delay Plot of HRV signals in (i) pre-meditative and (ii) meditative states under Chinese-chi meditation

Table III demonstrates that the value of the quantifying parameter $\mathrm{R}$ decreases during meditation in all cases under Chinese-chi meditation, while it increases in all cases under Kundalini yoga. In fact, the values of $\mathrm{R}$ in pre-meditative states are always greater than that of the meditative states under Chinese-chi meditation; whereas the values of $\mathrm{R}$ in pre-meditative states are always smaller than that of the meditative states under Kundalini yoga. So, for the purpose of distinction of these two different meditation techniques, 3D frequency-delay plot with proper frequency delay is most suitable and R may be taken as good quantifying parameters.

TABLE III

QUANTIFICATION TABLE OF 3D FREQUENCY-DELAY PLOT OF HRV SIGNALS IN PRE-MEDITATIVE AND MEDITATIVE STATES

\begin{tabular}{|c|c|c|c|c|c|c|c|c|}
\hline \multirow{2}{*}{ 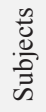 } & \multicolumn{4}{|c|}{ Pre-meditative States } & \multicolumn{4}{|c|}{ Meditative States } \\
\hline & SD1 & SD2 & SD3 & $\mathrm{R}$ & SD1 & SD2 & SD3 & $\mathrm{R}$ \\
\hline \multicolumn{9}{|c|}{ CHINESE - CHI MEDITATION } \\
\hline $\mathrm{c} 1$ & 0.87494 & 0.87358 & 0.59789 & 1.22979 & 0.839484 & 0.83856 & 0.5782 & 1.22459 \\
\hline c2 & 0.74884 & 0.76308 & 0.50651 & 1.26279 & 0.73776 & 0.73375 & 0.51748 & 1.20624 \\
\hline c3 & 0.76789 & 0.759589 & 0.50276 & 1.25002 & 0.823847 & 0.81703 & 0.56742 & 1.21581 \\
\hline $\mathrm{c} 4$ & 0.75385 & 0.765949 & 0.51233 & 1.25554 & 0.831385 & 0.82855 & 0.55787 & 1.24089 \\
\hline $\mathrm{c} 5$ & 0.80417 & 0.790928 & 0.53416 & 1.23212 & 0.897689 & 0.89759 & 0.62104 & 1.22261 \\
\hline c6 & 0.80117 & 0.800267 & 0.55362 & 1.22219 & 0.890694 & 0.88643 & 0.61745 & 1.21542 \\
\hline c7 & 0.75701 & 0.741045 & 0.49878 & 1.23232 & 0.751869 & 0.74948 & 0.52927 & 1.20644 \\
\hline $\mathrm{c} 8$ & 0.71789 & 0.711774 & 0.47106 & 1.25126 & 0.611144 & 0.61674 & 0.42316 & 1.23330 \\
\hline \multicolumn{9}{|c|}{ KUNDALINI YOGA } \\
\hline y1 & 0.937103 & 0.934930 & 0.65878 & 1.20843 & 0.622553 & 0.62178 & 0.43369 & 1.21619 \\
\hline y2 & 0.914141 & 0.900827 & 0.62502 & 1.21336 & 0.619447 & 0.61809 & 0.42854 & 1.22006 \\
\hline y3 & 0.912866 & 0.914739 & 0.64064 & 1.21495 & 0.759536 & 0.75743 & 0.52769 & 1.21630 \\
\hline y4 & 1.385892 & 1.3731806 & 0.95659 & 1.21317 & 0.723196 & 0.72271 & 0.50644 & 1.21318 \\
\hline
\end{tabular}

\section{Limitations and Remedy for the proposed method}

As the effect of meditation is studied under a few numbers of cases, thus the resultant effect is limited and cannot be generalized. However, the data set cannot be enlarged due to non-availability of such data in the Physionet database, which is the only source in these cases. So, this problem is resolved in the current work by statistical hypothesis testing as stated in section 2.5. For this purpose, eight values of the quantifying parameter $R$ for each of the eight different subjects in premeditative and meditative states are considered as two samples denoted by $R_{1}$ and $R_{2}$, then arranged in two columns. Therefore, it is established that the means of the corresponding populations consisting of all such 
elements of $R_{1}$ and $R_{2}$ coming out of a large number of subjects do differ significantly. The existence of any significant difference ensures that at certain level of confidence, it is enough to consider small samples of the form $R_{1}$ and $R_{2}$ in order to differentiate between meditative and premeditative states for large set of subjects.

Towards this goal, the population variances equality is tested in premeditative and meditative states using the test statistic, which given by:

$$
\mathrm{F}=\frac{\mathrm{s}_{1}^{2}}{\mathrm{~s}_{2}^{2}}
$$

Where, $s_{1}^{2}$ and $s_{2}^{2}$ are the sample variances. In case of Chinesechi meditation, $\mathrm{s}_{1}^{2}=0.000187268$ and $\mathrm{s}_{2}^{2}=0.000131178$. Therefore, $\mathrm{F}=1.427595<\mathrm{F}_{0.05(1), 7,7}=3.79$. Consequently, $\mathrm{H}_{0}$ holds and hence $\sigma_{1}^{2}=\sigma_{2}^{2}$. So, it is justified to apply student's t-test.

Meanwhile, the Student's $t$-test is performed to test the equality of population means in pre-meditative and meditative states as described in section 2.5.2, where $\mathbf{n}_{1}=\mathbf{n}_{2}=8$ and $v_{1}=7, v_{2}=7$, thus:

$$
s_{p}=\frac{\sum_{i} S S_{i}}{\sum_{i} v_{i}}=\frac{\sum_{i} v_{i} S_{i}^{2}}{\sum_{i} v_{i}}=0.017845056
$$

Where, $\overline{R_{1}}=1.242003657$ and $\overline{R_{2}}=1.220663651$, hence the test statistics is given by:

$$
\begin{aligned}
t & =\frac{\overline{\mathrm{R}}_{1}-\overline{\mathrm{R}}_{2}}{s_{p} \sqrt{\left(\frac{1}{\mathrm{n}_{1}}+\frac{1}{\mathrm{n}_{2}}\right)}}=\frac{0.02134}{0.008923} \\
& =2.39169955>\mathrm{t}_{0.05(2), 14}=1.76
\end{aligned}
$$

Therefore, the alternative hypothesis $\mathrm{H}_{\mathrm{A}}$ holds as well as a significant difference between the population means of pre-meditative and meditative states under Chinese-chi meditation is exist.

Similarly, for Kundalini yoga, ${ }_{1}^{2}=0.0000059332$ and $\mathrm{s}_{2}^{2}=0.00000595738$. Therefore, $\mathrm{F}=0.99593949$ is less than $\mathrm{F}$ $(0.05(2), 3,3)=15.4$; Hence $H_{0}$ holds. So, $\sigma_{1}^{2}=\sigma_{2}^{2}$ to perform the Student's t-test as follows:

$$
\mathrm{n}_{1}=\mathrm{n}_{2}=4, \overline{\mathrm{X}}=1.21247765, \overline{\mathrm{Y}}=1.216435246, \quad \text { thus }
$$

$\mathrm{S}_{\mathrm{p}}=0.0028155$ and the $\mathrm{t}$-test value will be:

$\mathrm{t}=4.82765986>\mathrm{t}(0.05(2), 6)=2.447$. Therefore, the alternative hypothesis $\mathrm{H}_{\mathrm{A}}$ holds and there is significant difference between the population means of pre-meditative and meditative states under Kundalini yoga.

From the preceding results, it is confirmed that the same trend in the results is observed even if the present work was carried out for larger sample size in both cases of Chinese-chi meditation and Kundalini yoga. The decrease of the quantifying parameter $(\mathrm{R})$ for each of the subjects in meditative states under Chinese-chi meditation, and the increase in each cases of Kundalini yoga indicates the impact effect of the chi meditation over the Kundalini yoga on the HRV. This establishes that the type of change is depending on the two different meditation techniques.

\section{CONCLUSION}

Meditation has a very strong effect on ANS and the type of effect is different for different mediation techniques. However, a very few attempts have been performed to mathematically differentiate the different meditation techniques. In the present study, the effect of Chinese-chi meditation and Kundalini yoga on the ANS has been studied towards distinguishing these two meditation techniques through the notion of 3D frequency-delay plots [26]. For this purpose, HRV signals in pre-meditative and meditative states of the persons practising Chinese-chi meditation and Kundalini yoga are obtained.

Since, time domain analysis fails to distinguish the aforesaid meditation techniques, the notion of 3D frequency-delay plot is applied. It has been observed that the value of the quantifying parameter (R) decreases for each of the subjects in meditative states under Chinesechi meditation, while it increases in each cases of Kundalini yoga. This not only establishes that the change in energy dynamics has taken place during meditation under both of Chinese-chi meditation and Kundalini yoga, but also it shows that the type of change is different for the two different meditation techniques.

Since, the samples are of small sizes, the results are substantiated by the statistical t-test. Thus, it may be concluded that the Chinesechi meditation and Kundalini yoga produce different types of changes in ANS. This changing pattern clearly distinguishes the aforesaid meditation techniques.

\section{REFERENCES}

[1] R.A. Baer, "Mindfulness training as a clinical intervention: A conceptual and empirical review," Clin. Psychol. Sci. Pract., vol. 10, pp. 125-143, 2003.

[2] M. B. Ospina, T. K. Bond, M. Karkhaneh, L. Tjosvold, B. Vandermeer, Y. Liang, L. Bialy, N. Hooton, N. Buscemi, D.M. Dryden, T. P. Klassen, "Meditation practices for health: state of the research," Evid. Rep. Technol. Assess (Full Rep), vol. 115, pp. 1-263, 2007.

[3] H.C. Lou, T.W. Kjaer, L. Friberg, G. Wildschiodtz, S. A Holm, "15O-H2OPET study of meditation and the resting state of normal consciousness," Hum. Brain Mapp., vol. 7, no.2, pp. 98-105, 1999.

[4] A. Newberg, A. Alavi, M. Baime, M. Pourdehnad, J Santanna, E. D. Aquili, "The measurement of regional cerebral blood flow during the complex cognitive task of meditation: A preliminary SPECT study," Psychiatr. Res., vol. 106, no. 2, pp. 113-122, 2001.

[5] M. M. S. Ahuja, M. G. Karmarkar, S. Reddy, TSH, LH, "Cortisol response to TRH and LH-RH and insulin hypoglycaemia in subjects practising transcendental meditation," Ind. J. Med. Res., 74, pp. 715, 1981.

[6] T. K. Akers, D. M. Tucker, R. S. Roth, J. S. VIDILOFF, "Personality correlates of EEG change during meditation," Psychological Reports, vol. 40, 1977.

[7] Y. Akishige, "Psychological studies on Zen," Bull. Fac. Lit. Kyushu Univ. (Japan), 5, 1968.

[8] I. B. Albert, B. McNeece, "The reported sleep characteristics of meditators and non-meditators," Bull. Psychon. Soc., vol. 3, 1974.

[9] C. Y. Liu, C. C. Wei, P. C. Lo, "Variation analysis of the sphygmogram to assess the cardiovascular system under meditation," Evid. Based Complement Alternat. Med., vol. 6, 2009.

[10] P. Lehrer, Y. Sasaki, Y. Saito, "Zazen and cardiac variability," Psychosom. Med. Vol. 61,1999.

[11] C. K. Peng, I. C. Henry, J. E. Mietus, J. M. Hausdorff, G. Khalsa, H. Benson, A. L. Goldberger, "Heart rate dynamics during three forms of meditations," Int. J. Cardiol., vol. 95, 2004. 
[12] C. K. Peng, J. E. Mietus, Y. Lie, G. Khalsa, P. S. Douglas, H. Benson, A. L. Goldberger, "Exaggerated heart rate oscillations during two meditation techniques," Int. J. Cardiol., vol. 70, 1999.

[13] D.P. Goswami, D.N. Tibarewala, D.K. Bhattacharya, "Analysis of heart rate variability signal in meditation using second-order difference plot," $J$. Appl. Phys., vol. 109, 2011.

[14] Y.H. Shiau, "Detecting Well-Harmonized Homeostasis in Heart Rate Fluctuations," BMEI, vol. 2, pp.399-403, 2008.

[15] A. Dey, S. Mukherjee, S. K. Palit, D. K. Bhattacharya, D. N. Tibarewala, "A new technique for the classification of pre-meditative and meditative states," Proc. of the International Conf. ICCIA, Kolkata, India, pp. $26-$ 28, 2010.

[16] M. Toichi, T. Sugiura, T. Murai, A. Sengoku, "A new method of assessing cardiac autonomic function and its comparison with spectral analysis and coefficient of variation of r-r interval," J. Auton. Nerv. Syst., vol. 62, pp. 79-82, 1997

[17] M. P. Tulppo, T. H. Makikallio, T. E. S. Takala, T. Seppanen, H. V. Huikuri " "Quantitative beat-to-beat analysis of heart rate dynamics during exercise," Am. J. Physiol., vol. 271, pp. 244-252, 1996.

[18] M. P. Tulppo, T. H. Makikallio, T. Seppanen, J. K. E. Airaksinen, H. V. Huikuri, "Heart rate dynamics during accentuated sympathovagal interaction," Am. J. Physiol., vol. 247, pp.810-816, 1998.

[19] J. Hayano, H. Takahash, T. Toriyama, S. Mukai, A. Okada, S. Sakata, A, Yamada, N. Ohte, H. Kawahara, "Prognostic value of heart rate variability during long term follow-up in chronic haemodialysis patients with endstage renal disease," Nephrol. Dial. Transplant., vol.14, pp. 1480-1488, 1999.

[20] Heart Rate Variability: An Indicator of Autonomic Function and Physiological Coherence, Institute of Heart Math, 2003. Available http:// www.heartmath.org/research/science-of-the-heart/soh_13.html.

[21] B. Irfan, A. E. Metin, K. Dayimi, T. Muhsin, K. Osman, M. Mehmet, B. E.Ozlem, B. Yelda, "Cigarette smoking and heart rate variability: dynamic influence of parasympathetic and sympathetic maneuvers, "Ann. Noninv. Electrocardiol., vol. 10, pp. 324-329, 2005.

[22] U. R. Acharya, K. P. Joseph, N. Kannathal, L. C. Min, J. S. Suri, “Advances in Cardiac Signal Processing: Heart Rate Variability," Springer, New York, pp. 121-165, 2007.

[23] C. K. Peng, J. E. Mietus, Y. Liu, G. Khalsa, P. S. Douglas, H. Benson, A. L. Goldberger, "Exaggerated heart rate oscillations during two meditation techniques," International journal of cardiology, vol. 70, no. 2, pp. 101$107,1999$.

[24] G. Kheder, A. Kachouri, R. Taleb, M. Ben Messaoud, M. Samet, "Feature extraction by wavelet transforms to analyze the heart rate variability during two meditation techniques," Advances in Numerical Methods, pp. 379-387, Springer US, 2009.

[25] G. Kheder, A. Kachouri, M. B. Massoued, M. Samet, "Heart rate variability analysis using threshold of wavelet package coefficients," International Journal on Computer Science and Engineering, Vol. 1, no. 3, pp. 131136, 2009.

[26] S. Mukherjee, S. K. Palit, "A New Scientific Study towards Distinction of ECG Signals of a Normal healthy person and of a Congestive Heart Failure Patient," J. Inter. Acad. Phys. Sci,. vol.15, no. 4, pp. 413-433, 2011.

[27] J. Piskorski, "Filtering Poincaré plots," Comp. Methods in Sci. \& Tech., vol. 11, 2005.

[28] M. Brennan, M. Palaniswami, P. Kamen, "Do existing measures of Poincaré plot geometry reflect nonlinear features of heart rate variability?," IEEE Trans. Biomed. Eng., vol 48, 2001.

[29] A. Voss, S. Schulz, R. Schroeder, M. Baumert, P. Caminal, "Methods derived from nonlinear dynamics for analysing heart rate variability," Phil. Trans. R Soc., pp. 277-296, 2009.

[30] C. Lerma, O. Infante, H. P. Grovas, M. V. Jose'," Poincare' plot indexes of heart rate variability capture dynamic adaptations after haemodialysis in chronic renal failure patients," Clin. Physiol. \& Func. Im., vol.23, pp. 72-80, 2003.

[31] M. Brennan, M. Palaniswami, P. Kamen, "Poincare' plot interpretation using a physiological model of HRV based on a network of oscillators," Am. J. Physiol. Heart Circ. Physiol., vol. 283, pp. H1873-H1886, 2002.

[32] J. Haaksma, J. Brouwer, W.A. Dijk, W.R.M. Dassen, D.J.V. Veldhuisen, "The dimension of 2D and 3D Poincaré plots obtained from 24 hours ECG registrations," IEEE Comp. in Cardiol., vol. 29, pp. 453-456,2002.

[33] A. S. Khaled, I. O. Mohamed, A. S. A. Mohamed, "Employing TimeDomain Methods and Poincaré Plot of Heart Rate Variability Signals to Detect Congestive Heart Failure," BIME J., vol. 6, no.1, 2006.

[34] S. Mensing, J. Limberis, G. Gintant, A. Safer, "A Novel Method for Poincaré Plot Shape Quantification Demonstrates Cardiac Tissue Repolarization Inhomogeneities Induced by Drugs," IEEE Comp. in Cardiol., vol. 35, 2008.

[35] M. Weeks, "Digital Signal Processing," Infinity Science Press LLC, Massachusetts, 2007.

[36] J. H. Zar, "Biostatistical Analysis," Pearson Education, 2006.

[37] A. L. Goldberger, L. A. N. Amaral, L. Glass, J. M. Hausdorff, P. Ch. Ivanov, R. G. Mark, J. E. Mietus, G. B. Moody, C. K. Peng, H. E. Stanley, "Physiobank, physiotoolkit, and physionet components of a new research resource for complex physiologic signals," Circul., vol. 101, no.23, 2000.

[38] G. P. Williams, "Chaos Theory Tamed," Joseph Henry Press, Washington, D.C., 1997.

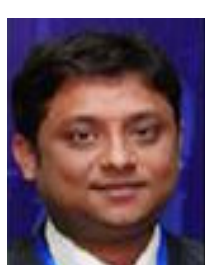

Dr.Anilesh Dey was born in West Bengal, India in 1977. He received the B.E in Electronics from Nagpur University and M.Tech.(Gold-Medallist) in Instrumentation and Control Engineering from Calcutta university and received $\mathrm{PhD}$. from Jadavpur University. He is working as Associate Professor and H.O.D of Electronics and Communication Engineering at The Assam Kaziranga University, Assam. $\mathrm{He}$ is author or co-author of more than 40 scientific papers in international/national journals and proceedings of the conferences with reviewing committee. His research topics nonlinear time series analysis, time and frequency domain analysis of bio- medical and music signals, effect of music in autonomic and central nervous system.

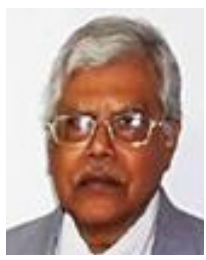

Prof.(Dr.)D. K. Bhattacharya was born in West Bengal, India in 1943. He is a retired Professor and Head in the department of Pure Mathematics University of Calcutta, India. He is presently an UGC Emeritus Fellow; prior to this he was an AICTE Emeritus Fellow of Govt. of India. $\mathrm{He}$ had his undergraduate, postgraduate and doctoral duty from the University of Calcutta. He has a long teaching experience of forty six years; he has supervised many Ph.D. students in Pure and Applied Mathematics. He is author or co-author of about 100 scientific papers in international /national journals and proceedings of the conferences with reviewing committee. His expertise is in Mathematical modeling and optimal control. His present interest is in application of Mathematics in Biology and Medicine including Bio-informatics.

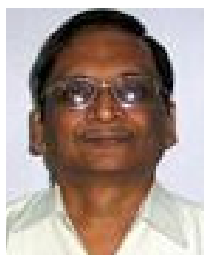

Prof.(Dr.)D.N. Tibarewala was born in Kolkata, December 1951. He is presently a Professor of Biomedical Engineering and formerly was Director in the School of Bioscience \& Engineering at Jadavpur University, Kolkata, India, he did his BSc (Honours) in 1971, and B. Tech (Applied Physics) in 1974 from the Calcutta University, India. He was admitted to the Ph.D. (Tech) degree of the same University in 1980. Having professional, academic and research experience of more than 30 years, Dr. Tibarewala has contributed about 200 research papers in the areas of Rehabilitation Technology, Biomedical Instrumentation and, related branches of Biomedical Engineering.

Amira S. Ashour, PhD., is an Assistant Professor and Vice Chair of Computers Engineering Department, Computers and Information Technology College, Taif University, KSA. She has been the vice chair of CS department, CIT college, Taif University, KSA for 5 years. She is in the Electronics and Electrical Communications Engineering, Faculty of Engineering, Tanta University, Egypt. She received her PhD in the Smart Antenna (2005) from the Electronics and Electrical Communications Engineering, Tanta University, Egypt. Her research interests include: image processing, Medical imaging, Machine learning, Biomedical Systems, Pattern recognition, Signal/image/video processing, Image analysis, Computer vision, and Optimization. She has 3 books and about 50 published journal papers. She is the Editor-in-Chief for the International Journal of Synthetic Emotions (IJSE), IGI Global, US. She is an Associate Editor for the IJRSDA, IGI Global, US as well as the IJACI, IGI Global, US. She is an Editorial Board Memberof the International Journal of Image Mining (IJIM), Inderscience. 


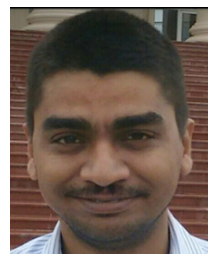

Nilanjan Dey, PhD., is an Asst. Professor in the Department of Information Technology in Techno India College of Technology, Rajarhat, Kolkata, India. He holds an honorary position of Visiting Scientist at Global Biomedical Technologies Inc., CA, USA and Research Scientist of Laboratory of Applied Mathematical Modeling in Human Physiology, Territorial Organization OfSgientifig and Engineering Unions, BULGARIA, Associate Researcher of Laboratoire RIADI, University of Manouba, TUNISIA. He is the Editor-in-Chief of International Journal of Ambient Computing and Intelligence (IGI Global), US, International Journal of Rough Sets and Data Analysis (IGI Global), US, and the International Journal of Synthetic Emotions (IJSE), IGI Global, US. He is Series Editor of Advances in Geospatial Technologies (AGT) Book Series, (IGI Global), US, Executive Editor of International Journal of Image Mining (IJIM), Inderscience, Regional Editor-Asia of International Journal of Intelligent Engineering Informatics (IJIEI), Inderscience and Associated Editor of International Journal of Service Science, Management, Engineering, and Technology, IGI Global. His research interests include: Medical Imaging, Soft computing, Data mining, Machine learning, Rough set, Mathematical Modeling and Computer Simulation, Modeling of Biomedical Systems, Robotics and Systems, Information Hiding, Security, Computer Aided Diagnosis, Atherosclerosis. He has 8 books and 170 international conferences and journal papers. He is a life member of IE, UACEE, ISOC etc. https://sites. google.com/site/nilanjandeyprofile/

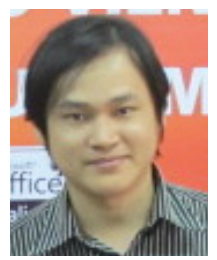

Dac-Nhuong Le has a MSc and Ph.D. in computer science from Vietnam National University, Vietnam in 2009 and 2015, respectively. He is Deputy-Head of Faculty of Information Technology, Haiphong University, Vietnam. Presently, he is also the Deputy-Cheif of Department of Educational Testing and Quality Assurance, Vice-Director of Information Technology Apply Center in the same university. $\mathrm{He}$ is a research scientist of R\&D Center of Visualization \& Simulation in, Duytan University, Danang, Vietnam. He has published numerous research articles in reputed international conferences, journals and online book chapters contributions. Currently his research interests are evaluation computing and approximate algorithms, network communication, security and vulnerability, network performance analysis and simulation, cloud computing, medical imaging.

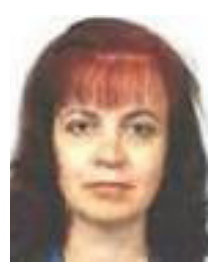

Evgeniya Gospodinova is an Assistant Professor of computer systems engineering at Institute of Systems Engineering and Robotics of Bulgarian Academy of Sciences. She received a M.Sc. degree in Microelectronics from the Department of Electronics at the Technical University of Gabrovo, Bulgaria and Ph.D. degree from the Central Laboratory of Mechatronics and Instrumentation of Bulgarian Academy of Sciences in 2009. The major fields of professional and scientific research interests include digital image processing, computer networks and communications, special instruments for information exchange, fractal modeling and analysis in traffic engineering and investigation of Heart Rate Variability of digital ECG signals. She is a member of the National Union of Automatics and Informatics.

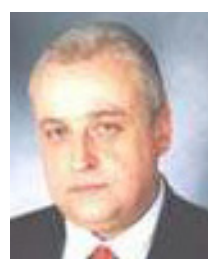

Mitko Gospodinova is an Assosiate Professor of computer systems engineering at Institute of Systems Engineering and Robotics of Bulgarian Academy of Sciences. He received a M.Sc. degree in Microelectronics from the Department of Electronics at the Technical University of Gabrovo, Bulgaria and Ph.D. degree from the Department of Computer Sciences at the Saint-Petersburg State Electrotechnical University, Russia in 1985. The major fields of professional and scientific research interests include digital image processing, computer networks and communications, analysis and design of electronic systems, automation of biomedical research, special instruments for information exchange, fractal modeling and analysis of self-similarity in traffic processes and biomedical systems. He is a member of the National Union of Automatics and Informatics. 